\title{
BMJ Open Quality Deprescribing for all: a narrative review identifying inappropriate polypharmacy for all ages in hospital settings
}

Ali Elbeddini (D) , ${ }^{1}$ Monakshi Sawhney, ${ }^{2}$ Yasamin Tayefehchamani, ${ }^{3}$ Zekiye Yilmaz, ${ }^{4}$ Ahmed Elshahawi, ${ }^{5}$ Josiah Josh Villegas, ${ }^{6}$ Janelyn Dela Cruz ${ }^{7}$

To cite: Elbeddini A,

Sawhney M, Tayefehchamani Y, et al. Deprescribing for all: a narrative review identifying inappropriate polypharmacy for all ages in hospital settings. BMJ Open Quality 2021;10:e001509. doi:10.1136/ bmjoq-2021-001509

- Additional online supplemental material is published online only. To view, please visit the journal online (http://dx.doi.org/10.1136/ bmjoq-2021-001509).

Received 29 March 2021 Accepted 26 June 2021

Check for updates

(C) Author(s) (or their employer(s)) 2021. Re-use permitted under CC BY-NC. No commercial re-use. See rights and permissions. Published by BMJ.

For numbered affiliations see end of article.

Correspondence to

Dr Ali Elbeddini;

Elbeddini.a@gmail.com

\section{INTRODUCTION}

The most common definition of polypharmacy is the concurrent use of 5 or more nonprescription and prescription medications by a single individual. ${ }^{1}$ There may be some instances of appropriate polypharmacy where therapy is optimised; however, for most cases, many medications may be unnecessary and even harmful. ${ }^{2}$ Polypharmacy has become a growing concern especially in ageing and at-risk populations due to its association with increased adverse drug events (ADE), drug interactions and decreased medication adherence. $^{3}{ }^{4}$ To reduce the incidence of polypharmacy and its consequences, healthcare providers have turned to deprescribing, the systematic process of identifying inappropriate medications to discontinue or taper down. Medications are inappropriate when they are no longer indicated or effective, and their existing or potential harms outweigh the benefits within the context of a patient's treatment goals, current level of functioning, life expectancy, values and preferences.

The majority of discussion and research surrounding deprescribing has focused on older adults largely due to the prevalence of polypharmacy in this population. Canadian data from 2016 show that about one in four elderly patients were prescribed 10 or more drug classes, the most common being statins, ACE inhibitors and proton-pump inhibitors. In addition, almost half (49.4\%) were prescribed at least one medication from the Beer's list, and $18 \%$ were prescribed multiple drugs from the list (online supplemental abstract 1$).^{5}$ The abundance of research data in geriatrics have led to the development of deprescribing guidelines and algorithms for practitioners to use. Although older adults are more sensitive to the side effects of medications and disproportionately experience medication-related harms, it would be valuable to investigate opportunities for deprescribing in patients under the age of 65 with multiple comorbidities and polypharmacy who may also benefit from the process. ${ }^{67}$

This article highlights the importance of deprescribing for patients across different age groups. Age alone should not be used as a criteria to start deprescribing and instead it should be offered for individuals at high risk of polypharmacy and where therapy is no longer appropriate. Beyond advanced age, there are many other risk factors that have been associated with increased polypharmacy exposure including: female gender, low education level, low income, number of hospitalisations, multiple comorbidities, multiple prescribers and high healthcare utilisation. ${ }^{89}$ These additional risk factors illustrate the many avenues of future research that support deprescribing in other groups. Furthermore, by initiating deprescribing earlier in a patient's life, we can proactively prevent polypharmacy in the elderly and the medication-related harms that are often seen in this population. More research is required to gather evidence for potentially inappropriate medications (PIMs) and deprescribing strategies for adults aged 18-65.

\section{METHODS}

For the purpose of this article, a literature search was completed using PubMed and Embase databases with the search terms of "polypharmacy", "multimorbidity", "deprescribing", "epidemiology", "drug use", "young adults", "middle age", "elderly". The elderly population was included in our search in order to draw comparisons to younger adults. Inclusion criteria were patients age 18 and above. Exclusion criteria were patients below age of 18, patients being treated in intensive care units and patients with cancer. Patients with cancer were specifically excluded as there are other deprescribing algorithms designed for this specialised population. 


\section{DISCUSSION}

\section{Epidemiology of polypharmacy}

According to data collected from 2007 to 2011 by Statistics Canada, multiple drug use increases by age and the percentage of prescription medication use in patients aged $45-64$ is almost double $(55.1 \%)$ compared with those aged 25-44 (28.0\%). This percentage increases to $82.7 \%$ in the $65-79$ age group. When it comes to polypharmacy, less than $2 \%$ of patients aged 25-44 are using five or more medications but this increases to $11.4 \%$ in the $45-64$ age group and $29.9 \%$ for the $65-79$ age group (online supplemental abstract 2) ${ }^{10}$ In a systematic review focusing on barriers and facilitators to deprescribing, a common barrier identified was that many patients were less inclined to stop medications they have been taking for many years. Patients often felt they 'needed' these medications, making it difficult for care providers to propose deprescription. ${ }^{11}$ Seniors involved in the Eliminating Medications Through Patient Ownership of End Results (EMPOWER) trial that elected not to discontinue their long-term benzodiazepine use, indicated that their main reasons were due to discouragement by their physician or pharmacist $(33 \%)$ and fear of withdrawal symptoms $(25 \%) .{ }^{12}$ Over the years, as more medications are prescribed due to a natural increase in morbidities and these regimens become routine, patients slowly move towards polypharmacy and become more resistant to stopping the medications that have worked for them in the past but may no longer be indicated. This is known as legacy prescribing, where drugs prescribed for an intermediate amount of time becomes a part of long-term therapy and is not properly discontinued. ${ }^{13}$ Younger adults are more likely to be actively involved in the management of their medications in comparison to older adults, making them more suitable candidates for deprescribing. ${ }^{14} 15$ It is, therefore, important to regularly assess a patient's need for a medication irrespective of their age in order take on a more proactive approach to deprescription and the prevention of polypharmacy rather than a reactive approach that is only initiated later on in life.

\section{Multimorbidity in younger adults}

Polypharmacy is largely driven by multimorbidity, and although this affects older adults the most, there is still a large number of patients with multimorbidity younger than 65 years, particularly in poor populations. ${ }^{16}$ It is interesting to note that even though the prevalence of medication use differs between the adult age groups, all groups shared the most commonly used drug classes including: lipid-lowering drugs, ACE inhibitors and acidreducers. Other medications that are more unique in the younger adults include antidepressants and inhalers. ${ }^{10} 17$ As the ability to detect asymptomatic conditions improves, the occurrence of preventative prescribing in adults has also increased. ${ }^{13}$ This has led to a recent increase in prophylactic medications for age-related disorders such as cardiovascular disease and osteoporosis in this age group. ${ }^{18}$ Among patients aged $25-79$ years, medications were mainly used to treat different medical conditions such as cardiovascular disease, dyslipidaemia, acid reflux disorders, depression and pain. ${ }^{17}$ The epidemiology of medication use varies between age groups, so the definition of taking 'too many medications' should also change correspondingly. Since different age groups can experience polypharmacy for different indications, it is imperative to explore applying the practice of deprescribing in those age groups to target their unique needs.

\section{Applying deprescribing tools to the younger population}

References and tools commonly used to help identify PIM for deprescription include the START/STOPP Criteria as well as the Beer's Criteria tool. ${ }^{19}$ Similar but customised tools should also be developed for younger adults given the lack of information and research on deprescribing in this population and because strategies used to target the elderly population will be less applicable for other adult age groups. For example, limited life expectancy is a factor to consider when discontinuing statins in the elderly, but this may not apply to a middle-aged adult. Furthermore, antipsychotic deprescribing would be a more appropriate consideration for elderly patients with BPSD than for younger patients for whom the antipsychotic is likely indicated chronically for a psychiatric diagnosis rather than for dementia. ${ }^{20}$ The Bruyere Research Institute Deprescribing Guidelines Research team has developed helpful guidelines and tools for deprescribing with a focus on medication classes that are often over prescribed and rarely discontinued despite a lack of indication. These classes include proton pump inhibitors, antipsychotics and benzodiazepines, and the guidelines also describe evidence and recommendations for younger adults with certain indications. ${ }^{21}$ It may be of value to tailor more research towards deprescribing in adults between the ages of 18-65. Although this population may not yet have as prevalent of a concern with polypharmacy as older individuals, targeting classes of medications that can be deprescribed can be the first step to preventing future harm

\section{Identifying candidates for deprescribing}

The goal of deprescribing is to reduce the number of PIMs, which has been and still is an ongoing challenge for all patients, particularly older adults. By decreasing the number of PIMs, we can optimise the health benefits of our patient's medications and reduce medicationrelated adverse events even at an early stage. Identifying patients who benefit the most from deprescribing should become the standard instead of using age as a determining factor for targeting patients. If we reduce the number of inappropriate medications early in life, patients can avoid future negative consequences such as preventable adverse drug reactions (figure 1). ${ }^{22}$ Technology, like electronic medical records (eg, EPIC, Cerner), can be used to add clinical alerts into patient profiles to inform pharmacists when patients present 


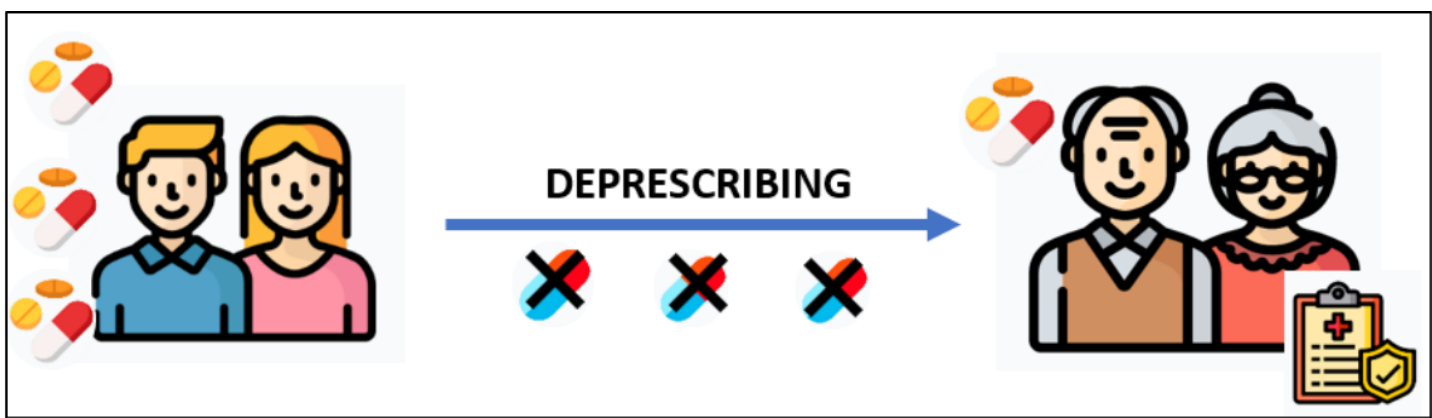

Figure 1 Proactive deprescribing in the younger adults helps prevent future polypharmacy-related adverse drug events.

with multiple comorbidities and medications that often do not have a clear indication such as benzodiazepines, antipsychotic and PPIs.

In hospital practice, candidates for deprescribing could be screened on admission during medication reconciliation and best possible medication histories. Pharmacists can ensure that each medication has a clear indication and its own place in a patient's medication regimen to improve overall health outcomes. An alert system should be developed in order to easily identify patients who are at high risk of polypharmacy or medications that can potentially be discontinued based on online health records (online supplemental abstract 3).

\section{Prompts for deprescribing}

\section{Medications for temporary symptoms}

Medications are often added to treat temporary symptoms and do not get reassessed or discontinued (table 1).
Medications used to treat a side effect resulting in a prescribing cascade

Medications are sometimes prescribed to treat a side effect of other medications resulting in a prescribing cascade. These cascades often lead to polypharmacy as well as increased adverse drug reactions, and they are often associated with increased treatment costs. ${ }^{23}{ }^{24}$ When a new symptom arises in a patient, it can be incorrectly diagnosed as a new medical condition instead of recognising that it is the result of an adverse reaction to an existing medication. This can lead to unnecessary prescribing of new medication(s) therefore resulting in other unnecessary drug interactions and side effects. ${ }^{25}$ For example, furosemide could be prescribed for transient leg swelling but not be discontinued, resulting in the development hypokalaemia, so the patient is prescribed a potassium supplement. This example is outlined in figure 2 and it illustrates how the failure to discontinue the original

Table 1 Examples of temporary medications continued as legacy prescriptions

Situation Risk of harm Example

Medications used during infections Potential misuse of the medication for a similar Continued use of salbutamol inhaler after for symptom relief symptom caused by another condition instead acute pulmonary infection of seeking care from a healthcare professional

Short term PPI therapy turns into chronic therapy

Long-term side effects of PPIs include impaired $\mathrm{B}_{12}$ absorption, hypomagnesaemia, increased risk of Clostridium difficile infection, community-acquired pneumonia ${ }^{41}$

Opioids during perioperative care
Benzodiazepines

Exposure to opioid toxicities, risk of developing dependence ${ }^{20}$

Associated with falls, memory disorders, dementia, functional impairment, daytime sedation, motor vehicle accidents ${ }^{42}$

Antipsychotics Increased risk of weight gain, dry mouth, dizziness, EPS, UTIs, cardiovascular adverse events $^{43}$

Antiepileptics
Significant drug-drug interactions (some are strong enzyme inducers); long-term side effects include bone density reduction, hyperlipidaemia, folate deficiency ${ }^{44}$
Chronic PPI use for indications such as GERD, mild-moderate oesophagitis, PUD caused by NSAID or Helicobacter pylori ${ }^{41}$ Continued postsurgery for non-cancer pain in an opioid-naïve patient

Used for depressive episodes or grief

Prescribed for insomnia or anxiety after moving into a new environment

Patient with childhood-onset epilepsy who is seizure free, but chronic antiepileptic use is not reassessed or discussed ${ }^{45}$

EPS, Extrapyramidal symptoms; GERD, Gastroesophageal reflux disease; NSAIDS, non-steroidal anti-inflammatory drug; PPI, proton-pump inhibitor; PUD, Peptic Ulcer disease; UTIs, Urinary Tract infections. 


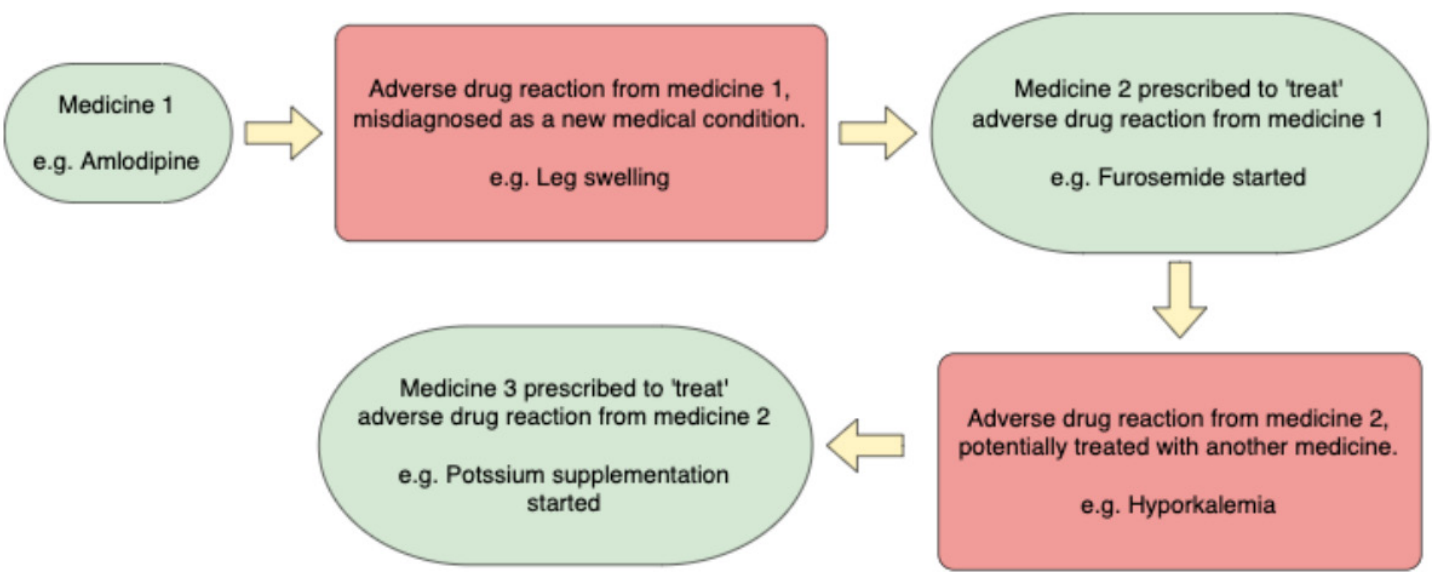

Figure 2 The prescribing cascade: adverse drug reaction from a medication, misdiagnosed as a new medical condition, which become treated with another medication leading to a cycle of prescribing for preventable harms.

medication after the resolution of a transient symptom resulted in the treatment of the medication's side effects. See online supplemental abstract 4 for other examples of prescribing cascades described in recent literature. ${ }^{26}$ In addition, online supplemental abstract 5 lists medications and notorious adverse effects which may be interpreted as a new medical condition and lead to the prescribing cascade.

A step-by-step strategy can be employed to prevent prescribing cascades. First, we can question if the patient's symptom(s) could have possibly originated as a side effect of an existing prescribed medication. It is important to rule-out a drug-induced adverse effect before proceeding further. The patient may also have trialled over-thecounter therapies and non-pharmacological treatments for this issue, so if the side effect is drug-induced, these self-treatments may also not be necessary. Second, assess if the offending medication is indicated for the patient or if it is no longer needed. Could it be switched to a safer alternative? Third, if that medication is still necessary, discuss the risks and benefits of continuing therapy with the healthcare team while including the patient in the process in order to educate and allow patients to make informed decisions. ${ }^{25}$

Prescribing cascades can be easily overlooked and although the elderly are more at risk, this concept can be applied to any patient with multiple chronic morbidities and medications. Again, addressing these issues early on can prevent adverse drug reactions and polypharmacy in a more vulnerable older population.

\section{Relationship between adherence and deprescribing}

It is important to distinguish 'deprescribing' from 'nonadherence' and 'non-compliance' because deprescribing is directed and supervised by healthcare professionals with the same level of expertise and attention that prescribing entails. ${ }^{27}$ It is also critical to remember that patient views and understanding of their medications have a direct relation to their willingness to adhere to or discontinue medications. All patients who struggle with medication adherence should be encouraged to ask their healthcare providers the five questions listed in online supplemental abstract 6 to prevent adverse effects of medications early on and to ask whether their medications can be reviewed to determine if any can be stopped or reduced. It can also be used by healthcare providers as a Teach-Back tool to confirm the patient's understanding of the reason for medication changes and discontinuations.

Deprescribing has the potential to improve patient adherence. Research has shown that an increased number of medications is associated with reduced adherence and that a reduced number of daily administrations leads to better adherence. ${ }^{28}{ }^{29}$ Considering that deprescribing can reduce the number of medications and the frequency of administrations, it could lead to overall improved adherence.

\section{Case study}

This patient is a 56-year-old man who was admitted to the hospital with community-acquired pneumonia. The pharmacist identified polypharmacy, with a regimen of $\mathrm{xx}$ medications, including xxx medications. The patient had difficulty managing his medications secondary to poor compliance and lack of awareness of the indications of his medications. He inquired about his medications but was instructed to ask his family physician instead and was offered a blister pack to improve compliance.

The patient has multiple comorbidities including hypertension, type 2 diabetes, peripheral neuropathy secondary to diabetes, urinary incontinence, frequent urinary tract infections over the past 6 months and chronic obstructive pulmonary disorder. He also has a history of falls due to dizziness spells and memory loss. The patient thinks his recent falls could be due to not taking some of his medications. The patient has poor diabetes control with an average A1C of $8.5 \%$ for the past year, leading to the worsening of his other medical conditions such as neuropathic pain.

\section{Medications list}

Insulin glargine 80 units two times a day, metformin $500 \mathrm{mg}$ three times a day with meals, linagliptin $5 \mathrm{mg}$ 
orally once daily, sildenafil $50 \mathrm{mg}$ tablet pro re nata (PRN), oxybutynin $5 \mathrm{mg}$ tablet three times a day, Acetylsalicylic acid (ASA) $81 \mathrm{mg}$ tablet once daily at bedtime, pregabalin $100 \mathrm{mg}$ three times a day, diclofenac emugel $2.32 \%$ for knee pain PRN, quinine sulfate $200 \mathrm{mg}$ two capsules at bedtime, dexlansoprazole $60 \mathrm{mg}$ capsule daily, hydrochlorothiazide $25 \mathrm{mg}$ once daily, perindopril $4 \mathrm{mg}$ once daily, diltiazem controlled delivery (CD) $180 \mathrm{mg}$ once daily, simvastatin $40 \mathrm{mg}$ tablet daily, salbutamol $100 \mu \mathrm{g}$ chlorofluorocarbon-free inhaler PRN, budesonide and formeterol 200/6 $\mu \mathrm{g}$ one inhalation two times a day, tiotropium $18 \mu \mathrm{g}$ one inhalation once daily, acetaminophen $650 \mathrm{mg}$ three times daily prn, blood glucose test strips, acetaminophen with codeine (8mg) 1-2 tablets every 8 hours PRN, salbutamol inhaler to use PRN for trouble breathing ( $\max 8$ puffs a day), ginseng two capsules a day for memory, hydrocortisone cream $1 \%$ for itchy skin and oxycodone/acetaminophen tablet 1-2 tabs every 6 hours PRN for breakthrough pain.

\section{Lifestyle and social factors}

The patient is obese with a body mass index of $32 \mathrm{~kg} / \mathrm{m}^{2}$. The patient smoked 1.5 packs a day and stopped using nicotine patches. The patient reports consuming 20 drinks of alcohol weekly. His neuropathic pain has limited his mobility and he can barely walk, which is particularly problematic as he lives alone in an apartment on the fifth floor. The patient is an accountant but has been off work for the past 9 months due to his medical conditions and has private insurance from his work.

\section{Intervention}

After a careful assessment of the patient's medication list and medication experience, the overall number of medicines was reduced from 15 to 10 (excluding devices), and dosing frequency was reduced from 15 to 7 (excluding PRN medicines).

\section{PHARMACOECONOMIC EVIDENCE ON DEPRESCRIBING}

Polypharmacy has been known to be associated with side effects, leading to adverse patient outcomes and even hospitalisations. More recent studies have also found a statistical relationship between polypharmacy and mortality. ${ }^{30} 31$ ADEs account for more morbidity and mortality than most chronic diseases, with death rates higher than many common cancers. ${ }^{32-34}$ A British hospital study found that in patients above age 16, 6.5\% of hospital admissions were due to an ADE, and in $80 \%$ of cases, the ADE was directly linked to the admission. The most commonly involved drugs were NSAIDs, diuretics and warfarin (online supplemental abstract 5 ) ${ }^{30}$ Recent data from the Canadian Institute for Health Information (CIHI) also shows more than half of ADE-related cases involved seniors, and that the higher the number of medications they took, the more likely they were to be hospitalised from an ADE. ${ }^{35}$ Therefore, recognising polypharmacy and initiating deprescribing can prevent inappropriate medication use as well as ADEs contributing to hospital admission. Medication reconciliations can ensure that a patient's medications are reviewed at each transition of care and it has a key role in reducing risk of ADEs as well as creating further opportunities for deprescribing.

In addition to improved clinical outcomes, deprescribing may also lead to reduced drug costs. ${ }^{36}$ As the population continues to age, the cost to the government of subsidising pharmaceuticals is increasing. Furthermore, several deprescribing interventions have established a decrease in drug costs. Kutner et al estimated that their intervention resulted in mean savings of US $\$ 716.46$ per participant based on the cost of the generic brand of statin within the mean follow-up period of 212.6 days during which the participants remained off the statin. ${ }^{37}$

In terms of cost to the healthcare system, the median hospital stay duration was 8 days and the average cost per hospital day was US $\$ 414$, contributing to an annual cost for these hospital admissions to be US\$ $\$ 847$ million. Extrapolating the data above, the average hospital stay would cost from US $\$ 3000$ to US $\$ 5000$. Given an estimate that $1 \%$ of hospital admissions involved ADEs due to PIMs and assuming a hospital engages in 15000 discharges a year 150 cases could be prevented with deprescribing. This would represent US $\$ 450000-$ US $\$ 750000$ in savings per year (US\$3000-US\$5000× 150 ). As a result, implementating deprescribing processes could result in significant savings to the healthcare system that can be used in other underfunded areas.

According to statistics from the CIHI, the median hospital stay duration in Canada for 2018-2019 was 7.5 days and the average cost per patient day in medical and surgical units was US\$511.1 which contributes to an annual direct cost to be $\$$ C6.515 billion. ${ }^{38}$ Extrapolating from the data above, the average hospital stay would cost approximately \$C3833. Given that an estimate of $6.5 \%$ of hospital admissions were due to an ADE and given that in Canada a total of 3086942 discharges occurred in 20182019 , the number of discharges attributed to ADE were $200651 .^{39}$ If these ADE were prevented with proactive deprescribing, a total of $\$ \mathrm{C} 769$ million of healthcare costs could be saved per year. In essence, implementing deprescribing processes could result in significant savings to the healthcare system which can be used in other underfunded areas.

\section{CONCLUSION}

WHO has highlighted inappropriate polypharmacy as one of the key focus areas of its Third Global Patient Safety Challenge, Medication Without Harm. ${ }^{40}$ Polypharmacy is not necessarily restricted to older adults. In fact, younger patients can also benefit from proactive deprescribing since it would prevent polypharmacy before it begins. In essence, this reduces the amount of legacy prescribing. Identifying PIM use is well established in the elderly, but more research is necessary to create similar strategies for the younger age groups. These strategies may target 
individual or a combination of consequential factors such as multiple comorbidities, high-risk medications, lack of benefit, or duplicate therapy. In clinical practice, healthcare providers can start deprescribing initiatives targeting patients with polypharmacy, some of which may be unnecessary or cause harm, and by doing so, lead to decreased risks of adverse effects and future hospitalisations.

\section{Author affiliations}

${ }^{1}$ Family Medicine, University of Ottawa, Ottawa, Ontario, Canada

${ }^{2}$ School of Nursing \& Department of Anesthesiology and Perioperative Medicine, Queen's University, Kingston, Ontario, Canada

${ }^{3}$ Clinical Pharmacy, University of Toronto Leslie Dan Faculty of Pharmacy, Toronto, Ontario, Canada

${ }^{4}$ Clinical Pharmacy- Head of department, Acibadem University, Istanbul, İstanbul, Turkey

${ }^{5}$ Assistant professor of Medicine, University of Toronto Faculty of Medicine, Toronto, Ontario, Canada

${ }^{6}$ Faculty of Pharmacy, University of Toronto Leslie Dan, Toronto, Ontario, Canada ${ }^{7}$ MD Candidate, Saint James School of Medicine - Saint Vincent and the Grenadines Campus, Arnos Vale, Saint George, Saint Vincent and the Grenadines

Twitter Ali Elbeddini @Dr. Ali Elbeddini

Acknowledgements We would like to thank the pharmacy team who supported in collecting the data.

Contributors All authors have agreed and approved the final draft.

Funding The authors have not declared a specific grant for this research from any funding agency in the public, commercial or not-for-profit sectors.

Competing interests None declared.

Patient and public involvement Patients and/or the public were not involved in the design, or conduct, or reporting, or dissemination plans of this research.

Patient consent for publication Not required.

Ethics approval Ethics approval was obtained from the Ethics Committee.

Provenance and peer review Not commissioned; externally peer reviewed.

Supplemental material This content has been supplied by the author(s). It has not been vetted by BMJ Publishing Group Limited (BMJ) and may not have been peer-reviewed. Any opinions or recommendations discussed are solely those of the author(s) and are not endorsed by BMJ. BMJ disclaims all liability and responsibility arising from any reliance placed on the content. Where the content includes any translated material, BMJ does not warrant the accuracy and reliability of the translations (including but not limited to local regulations, clinical guidelines, terminology, drug names and drug dosages), and is not responsible for any error and/or omissions arising from translation and adaptation or otherwise.

Open access This is an open access article distributed in accordance with the Creative Commons Attribution Non Commercial (CC BY-NC 4.0) license, which permits others to distribute, remix, adapt, build upon this work non-commercially, and license their derivative works on different terms, provided the original work is properly cited, appropriate credit is given, any changes made indicated, and the use is non-commercial. See: http://creativecommons.org/licenses/by-nc/4.0/.

ORCID iD

Ali Elbeddini http://orcid.org/0000-0002-3339-6203

\section{REFERENCES}

1 Masnoon N, Shakib S, Kalisch-Ellett L, et al. What is polypharmacy? A systematic review of definitions. BMC Geriatr 2017;17:230.

2 Khan N, Chattopadhyay K, Leonardi-Bee J, Incidence L-BJ. Incidence, prevalence, risk factors and health consequences of polypharmacy in adults in South Asia: a systematic review protocol. JBI Database System Rev Implement Rep 2019;17:2370-7.

3 Halli-Tierney AD, Scarbrough C, Carroll D. Polypharmacy: evaluating risks and deprescribing. Am Fam Physician 2019;100:32-8.

4 Mabuchi T, Hosomi K, Yokoyama S, et al. Polypharmacy in elderly patients in Japan: analysis of Japanese real-world databases. J Clin Pharm Ther 2020;45:991-6.
5 Government of Canada SC. Research Highlights on Health and Aging [Internet], 2016. Available: https://www150.statcan.gc.ca/n1/ pub/11-631-x/11-631-x2016001-eng.htm

6 Onder G, Marengoni A. Polypharmacy. JAMA 2017;318:1728.

$7 \mathrm{ClHI}$. Drug use among seniors in Canada. Available: https://www.cihi. $\mathrm{ca} / \mathrm{en} /$ drug-use-among-seniors-in-canada

8 Assari S, Bazargan M. Race/Ethnicity, socioeconomic status, and polypharmacy among older Americans. Pharmacy 2019;7:41.

9 Badawy NA, Labeeb SA, Alsamdan MF, et al. Prevalence and risk of polypharmacy among community-dwelling, elderly Kuwaiti patients. Med Princ Pract 2020;29:166-73.

10 Supplementary Appendix - ProQuest [Internet]. Available: https:// search.proquest.com/docview/873180468

11 Doherty AJ, Boland P, Reed J, et al. Barriers and facilitators to deprescribing in primary care: a systematic review. BJGP Open 2020;4:bjgpopen20X101096.

12 Tannenbaum C, Martin P, Tamblyn R, et al. Reduction of inappropriate benzodiazepine prescriptions among older adults through direct patient education: the empower cluster randomized trial. JAMA Intern Med 2014;174:890-8.

13 Walker LE, Mangoni AA. Avoiding harm from overprescribing: what are the challenges and how do we overcome them? Br J Clin Pharmacol 2021;87:6-8.

14 Straßner C, Steinhäuser J, Freund T, et al. German healthcare professionals' perspective on implementing recommendations about polypharmacy in general practice: a qualitative study. Fam Pract 2018;35:503-10.

15 Schöpf AC, von Hirschhausen M, Farin E, et al. Elderly patients' and GPs' perspectives of patient-GP communication concerning polypharmacy: a qualitative interview study. Prim Health Care Res Dev 2018;19:355-64.

16 Barnett K, Mercer SW, Norbury M, et al. Epidemiology of multimorbidity and implications for health care, research, and medical education: a cross-sectional study. Lancet 2012;380:37-43.

17 Association between polypharmacy and death: A systematic review and meta-analysis [Internet]. Available: https://www. areasaludbadajoz.com/index.php/doc-e-investigacion/bibliotecadel-area/biblioteca-de-enfermeria/556-association-betweenpolypharmacy-and-death-a-systematic-review-and-meta-analysis

18 Bennett F, Ferner R, Sofat R. Overprescribing and rational therapeutics: barriers to change and opportunities to improve. $\mathrm{Br} \mathrm{J}$ Clin Pharmacol 2021;87:34-8.

19 Saunders DJ. Adverse drug reactions as cause of admission to hospital: not all drugs that cause adverse reactions are actually prescribed by doctors. BMJ 2004;329:459.2.

20 Bjerre LM, Farrell B, Hogel M, et al. Deprescribing antipsychotics for behavioural and psychological symptoms of dementia and insomnia: evidence-based clinical practice guideline. Can Fam Physician 2018;64:17-27.

21 Deprescribing Guidelines and Algorithms [Internet]. Deprescribing. org. Available: https://deprescribing.org/resources/deprescribingguidelines-algorithms/

22 Motter FR, Fritzen JS, Hilmer SN, et al. Potentially inappropriate medication in the elderly: a systematic review of validated explicit criteria. Eur J Clin Pharmacol 2018;74:679-700.

23 Nguyen PV-Q, Spinelli C. Prescribing cascade in an elderly woman. Can Pharm J 2016;149:122-4.

24 Ponte M, Serra H, Wachs A, et al. A proposed new to evaluate it. Rev Med B Aires 2017;77:13-17.

25 Schachter SC. Determining when to stop antiepileptic drug treatment. Curr Opin Neurol 2018;31:211-5.

26 Hales CM, Servais J, Martin CB. Prescription drug use among adults aged 40-79 in the United States and Canada. NCHS 2019:11-12.

27 Reeve E, Thompson W, Farrell B. Deprescribing: a narrative review of the evidence and practical recommendations for recognizing opportunities and taking action. Eur J Intern Med 2017;38:3-11.

28 Chapman RH, Benner JS, Petrilla AA, et al. Predictors of adherence with antihypertensive and lipid-lowering therapy. Arch Intern Med 2005; $165: 1147-52$

29 Nieuwlaat R, Wilczynski N, Navarro T, et al. Interventions for enhancing medication adherence. Cochrane Database Syst Rev 2014:CD000011.

30 O'Mahony D, O'Sullivan D, Byrne S, et al. STOPP/START criteria for potentially inappropriate prescribing in older people: version 2. Age Ageing 2015;44:213-8.

31 Government of Canada SC. Prescription medication use by Canadians aged 6 to 79 - ARCHIVED [Internet], 2014. Available: https://www150.statcan.gc.ca/n1/en/catalogue/82-003X201400614032 
32 Lipska KJ, Ross JS, Wang Y, et al. National trends in US hospital admissions for hyperglycemia and hypoglycemia among Medicare beneficiaries, 1999 to 2011. JAMA Intern Med 2014;174:1116-24.

33 Lazarou J, Pomeranz BH, Corey PN. Incidence of adverse drug reactions in hospitalized patients: a meta-analysis of prospective studies. JAMA 1998;279:1200-5.

34 Malvezzi M, Bertuccio P, Levi F, et al. European cancer mortality predictions for the year 2013. Ann Oncol 2013;24:792-800.

35 Griebling TL. Re: American geriatrics Society 2019 updated AGS beers Criteria $₫$ for potentially inappropriate medication use in older adults. J Urol 2019;202:438.

36 Kemp A, Preen DB, Glover J, et al. How much do we spend on prescription medicines? out-of-pocket costs for patients in Australia and other OECD countries. Aust Health Rev 2011;35:341-9.

37 Kutner JS, Blatchford PJ, Taylor DH, et al. Safety and benefit of discontinuing statin therapy in the setting of advanced, lifelimiting illness: a randomized clinical trial. JAMA Intern Med 2015;175:691-700.

38 Canadian Institute for Health Information. Trends in hospital spending, 2005-2006 to 2018-2019 - data tables - series C: average direct cost per patient by selected functional centre. Ottawa, ON: $\mathrm{ClHI}, 2020$.

39 Canadian Institute for Health Information. Inpatient hospitalizations: volumes, length of stay and standardized rates. Ottawa, $\mathrm{OH}: \mathrm{ClHI}$, 2020.

40 WHO. WHO Global Patient Safety Challenge: Medication Without Harm [Internet]. Available: http://www.who.int/patientsafety/ medication-safety/medication-without-harm-brochure/en/

41 Maund E, Stuart B, Moore M, et al. Managing antidepressant discontinuation: a systematic review. Ann Fam Med 2019;17:52-60.

42 Gaitatzis A, Sander JW. The long-term safety of antiepileptic drugs. CNS Drugs 2013;27:435-55.

43 Hah JM, Bateman BT, Ratliff J, et al. Chronic opioid use after surgery: implications for perioperative management in the face of the opioid epidemic. Anesth Analg 2017;125:1733-40.

44 Pottie K, Thompson W, Davies S, et al. Deprescribing benzodiazepine receptor agonists: evidence-based clinical practice guideline. Can Fam Physician 2018;64:339-51.

45 Evolution of brain-computer interface: action potentials, local field potentials and electrocorticograms- ClinicalKey [Internet]. Available: https://www.clinicalkey.es/\#!/content/1-s2.0-S0959438810001789 